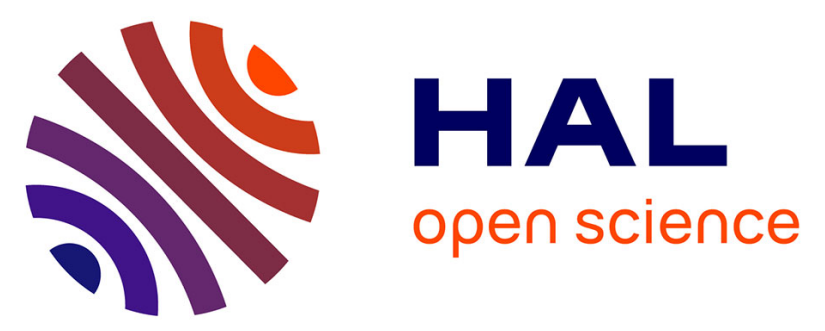

\title{
NONLINEAR OSCILLATIONS OF THE YATES-PARDEE-GOODWIN METABOLIC PATHWAY WITH COUPLING; THE ROLE OF EXTERIOR POOLS
}

Jean-Paul Morillon, Saïd Doubabi, Robert Costalat

\section{To cite this version:}

Jean-Paul Morillon, Saïd Doubabi, Robert Costalat. NONLINEAR OSCILLATIONS OF THE YATES-PARDEE-GOODWIN METABOLIC PATHWAY WITH COUPLING; THE ROLE OF EXTERIOR POOLS. Journal of Biological Systems, 1997, Volume 05, Issue 02, June 1997, pp.285-300. 10.1142/S0218339097000187 . hal-00967555

\section{HAL Id: hal-00967555 \\ https://hal.science/hal-00967555}

Submitted on 2 Apr 2014

HAL is a multi-disciplinary open access archive for the deposit and dissemination of scientific research documents, whether they are published or not. The documents may come from teaching and research institutions in France or abroad, or from public or private research centers.
L'archive ouverte pluridisciplinaire HAL, est destinée au dépôt et à la diffusion de documents scientifiques de niveau recherche, publiés ou non, émanant des établissements d'enseignement et de recherche français ou étrangers, des laboratoires publics ou privés. 
JOURNAL OF BIOLOGICAL SYSTEMS

\section{Nonlinear oscillations of the}

\section{Yates-Pardee-Goodwin metabolic pathway with coupling}

\section{The role of exterior pools}

J.-P. Morillon, S. Doubabi and R. Costalat

I.U.F.M. de Rouen, B.P. 18, 2, rue du Tronquet, 76131 Mont-Saint-Aignan Cédex 04, France.

Département de Physique, Faculté des Sciences, Guéliz, B.P. 618, Marrakech, Maroc.

CREARE-INSERM, Université Pierre et Marie Curie, Boite 23,

9, quai St-Bernard, 75252 Paris Cédex 05, France.

Correspondence to R. Costalat, CREARE-INSERM,

Université Pierre et Marie Curie, Boîte 23,

9, quai St-Bernard, 75252 Paris Cédex 05, France.

Tel. (1) 44.27.34.37 — Fax. (1) 44.27.34.38

E-mail: robert.costalat@snv.jussieu.fr 


\section{ABSTRACT}

An analytic method referred to as the harmonic balancing technique has been applied in order to study the effect of diffusion between compartments (coupling between pools) on the Yates-PardeeGoodwin metabolic pathway. The behaviour of the pathway is modelled on a set of coupled ordinary nonlinear differential equations. This set is transformed into a single feedback control loop with a nonlinear function and a linear filter. It is shown that coupling modifies the linear filter such that the frequency and the amplitude of the oscillations decrease. Further results are then obtained by numerical studies, and compared with the analytical approach and previous stability analysis of metabolic pathways.

Keywords: Metabolic pathways - Pools - Oscillations

- Compartments - Enzyme organization - Stability. 


\section{Introduction}

The most commonly encountered form of regulated biochemical pathway (or metabolic pathway), generally referred to as the Yates-Pardee or Goodwin metabolic pathway, consists of a single pathway of enzymatic reactions, where the last product inhibits the first enzyme (single loop negative feedback) $[7,20]$. A number of theoretical studies have been devoted to the Yates-PardeeGoodwin pathway, modelled on a system of nonlinear ordinary differential equations. Specifically, it was shown that $(i)$ the system can admit a unique steady state, stable or unstable, (ii) solutions initiated near the steady state, when it is stable, generally display damped oscillations, $(i i i)$ when the length of the metabolic pathway is increased, the stability domain of the steady state is decreased, and ( $i v)$ when the steady state is unstable, a stable limit cycle is often established [16,20].

These initial studies were concerned mainly with reactions occuring in a homogeneous milieu. More recently, several authors have emphasized the dramatic importance of the cell milieu heterogeneity for metabolic pathways dynamics [10,21]. For instance, Marmillot et al. [10] using a model where phosphofructokinase is partitioned between two phases, revealed the possibility of polarized wave phenomena which may be a basis for signal transmission in eukaryotic cells. From a different point of view, we showed that the parallel association of two Goodwin metabolic pathways, modelled on a set of nonlinear coupled first- 
order ordinary differential equations, can increase the stability domain of the unique steady state [14]. Further analytical and numerical studies of the stability of this system show that $(i)$ the association of two metabolic pathways [1], or, $(i i)$ the confinement of a metabolic pathway to a cellular compartment (enzyme organization), with exchange of matter with other compartments $[2,3]$, can lead to an increase of the rate of convergence towards the stable steady state. From a biological point of view, the results suggest that the exchange of matter between compartments (e.g. cells, organelles, ...) may be a source of stability for the cell metabolism.

The question arises whether this unusual property can be shown in the case of nonlinear oscillations: how coupling with exterior pools of metabolites modifies the behaviour of the oscillations of a Goodwin metabolic system.

In Section 2 the biological model is presented. In Section 3 some definitions and concepts of the harmonic balancing method are given. In Section 4 we apply this technique in order to compare the oscillations of the systems with or without coupling. In Section 5 numerical experiments are given, and in the last section some conclusions are submitted, in particular on the stability of steady state. 


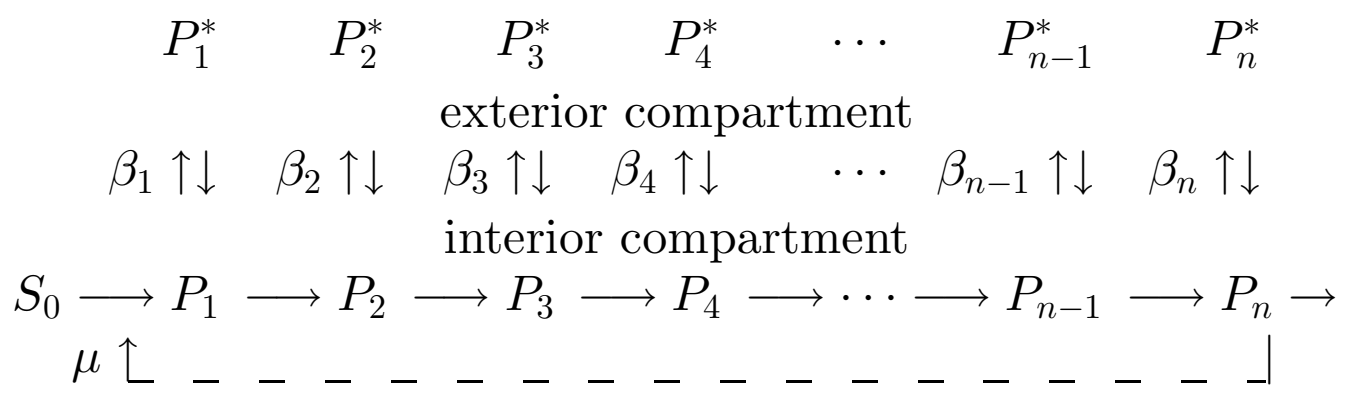

Fig. 1. Goodwin biochemical pathway with couplings

\section{The model with coupling}

We shall now investigate the oscillations of the Yates-Pardee or Goodwin metabolic pathway [7] by means of the harmonic balancing technique [16], with the addition of some passive diffusion coupling (see Fig. 1). More precisely, we assume that $(i)$ a cell or an organelle contains a Goodwin metabolic pathway [7]; $(i i)$ the metabolite $P_{i}, i=1, \ldots, n$, can exchange matter with the outside pool $P_{i}^{*}$ (for instance the same metabolite present in the cytosol) via passive diffusion, with a non-negative coefficient $\beta_{i}$. In this study, we shall assume that the compartment which contains the metabolic pathway (interior compartment) and the exterior compartment have the same volume.

Let us apply the laws of mass-balance and enzyme kinetics to this system, with passive diffusion between interior and exterior compartments.

Implementing the transformation of Walter $[16,20]$, the system can be mod- 
elled on the following set of differential equations that describe the time evolution of metabolic concentrations:

$$
\left\{\begin{array}{l}
\frac{d P_{1}}{d t}=f\left(P_{n}\right)-b_{1} P_{1}-\beta_{1}\left(P_{1}-P_{1}^{*}\right), \\
\frac{d P_{k}}{d t}=P_{k-1}-b_{k} P_{k}-\beta_{k}\left(P_{k}-P_{k}^{*}\right), \quad k=2, \ldots, n, \\
\frac{d P_{k}^{*}}{d t}=\quad \beta_{k}\left(P_{k}-P_{k}^{*}\right), \quad k=1, \ldots, n,
\end{array}\right.
$$

where $P_{i}$ and $P_{i}^{*}$ are the respective time-dependent concentrations of a given metabolite in the interior and the exterior compartments. The coefficient $b_{i}$ characterizes the positive kinetic constant of the reaction $P_{i} \rightarrow P_{i+1}$ in the metabolic pathway. The reaction function $f$ is given; in the Goodwin metabolic pathway, $f$ describes the allosteric feedback inhibition of the first reaction by the last product, and can be written as follows:

$$
f\left(P_{n}\right)=\frac{1}{1+\left(P_{n}\right)^{\mu}} .
$$

where $\mu$ is a positive integer. Finally, we assume that association with exterior pools results in passive diffusion, with constant non-negative coefficients $\beta_{i}$. If the value of $\beta_{i}$ is always zero, the metabolic pathway is said to be isolated or non-associated.

The existence and uniqueness of the positive steady state are proved in [14]. The results of the linear stability analysis of the system at the neighborhood 
of the unique steady state can be derived by both analytical ${ }^{1}$ and numerical ${ }^{2}$ studies for some values of the parameters of the model (see [14]). Apart from the fact that a systematic study of the local properties derived by linearization may rapidly become cumbersome, the question of discerning whether a system possesses a stable positive steady state or a limit cycle remains to be answered. However, existence of stable oscillations for a Goodwin system without coupling has been proved in [8].

\section{First order harmonic balancing technique}

The differential equations (2.1) are nonlinear, have large dimensions and contain a large number of reaction constants which must be treated as independent parameters. The harmonic balancing technique presented here can successfully analyse a large class of biochemical oscillators throughout the associated parameter space and for arbitrary dimension (see, for instance, [15]), in particular when the function $f$ of the feedback loop is positive, and monotonically decreasing with its argument.

\footnotetext{
${ }^{1}$ Using the criterion of Liénard and Chipart.

${ }^{2}$ By computing the eigenvalues of jacobian matrix with scientific software such as MATLAB.
} 


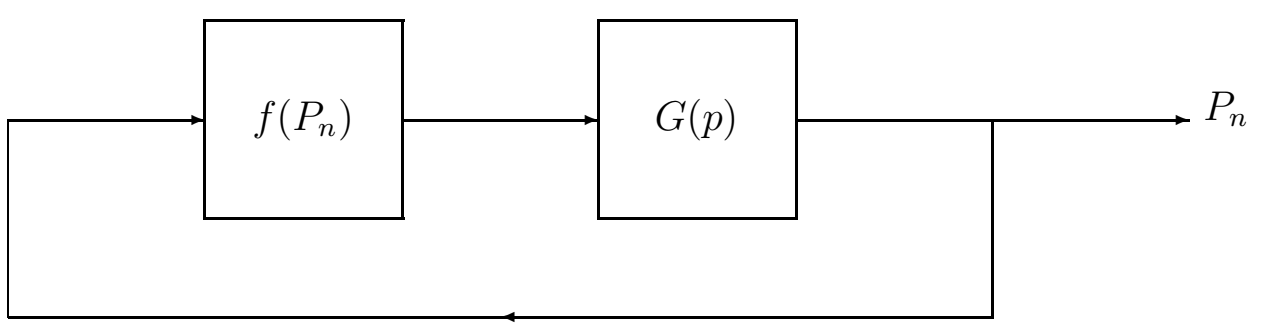

Fig. 2. Single feedback loop with a nonlinear function $f$ and a linear filter $G(p)$

\subsection{Definitions}

This mathematical method $[11,5,9]$ approximates the output $P_{n}$ and the nonlinear element $f\left(P_{n}\right)$ by a partial sum of their Fourier series:

$$
\begin{aligned}
P_{n}(t) & =\mathcal{R} e\left(\sum_{k=0}^{p} x_{k} \exp (i k \omega t)\right), \\
f\left(P_{n}(t)\right) & =\mathcal{R} e\left(\sum_{k=0}^{p} y_{k} \exp (i k \omega t)\right),
\end{aligned}
$$

where the coefficients $y_{k}$ verify:

$$
\begin{aligned}
& y_{0}=\frac{1}{2 \pi} \int_{0}^{2 \pi} f\left(\mathcal{R} e\left(\sum_{l=0}^{p} x_{l} \exp (i l t)\right)\right) d t, \\
& y_{k}=\frac{1}{\pi} \int_{0}^{2 \pi} f\left(\mathcal{R} e\left(\sum_{l=0}^{p} x_{l} \exp (i l t)\right)\right) \exp (-i k t) d t, \quad 1 \leq k \leq p .
\end{aligned}
$$

Using the loop's input-output equation :

$$
P_{n}=G f\left(P_{n}\right)
$$


where $G$ is the linear filter so that:

$G f\left(P_{n}\right)=\mathcal{R} e\left(G(0) y_{0}+G(i \omega) y_{1} \exp (i \omega t)+\ldots+G(i p \omega) y_{p} \exp (i p \omega t)\right)$,

we obtain $p+1$ balance equations :

$$
G(i k \omega) y_{k}=x_{k}, \quad 0 \leq k \leq p
$$

Then, an approximation to $P_{n}(t)$ is obtained by solving these equations. In order to justify the truncation at $p=1$, we first say that for all but the most trivial nonlinearities the application of this approximation becomes analytically impractical as $p$ increases. Furthermore, the coefficient $x_{k}$ decreases in magnitude with increasing $k$ since $f\left(P_{n}\right)$ looks much like a step function for $p \geq 2$, and for a square wave: $\left|x_{k}\right|=\mathrm{O}(1 / k)$ (see, for instance, [17, Appendix B]). Also, $|G(i k \omega)|$ decreases rapidly for increasing $k$, and tends to filter out higher harmonics of $f\left(P_{n}\right)$ with $f$ of the specific form (2.2). An example of the breakdown of the first order approximation is given in [11, Section 4]: the 3rd order approximation is then applied to give the effect of a cubic nonlinearity [11]. Since the Fourier series of the function $f$ defined by (2.2) converges rapidly and $G(p)$ is an extreme low pass filter, the first order approximation:

$$
P_{n}(t)=x_{0}+x_{1} \cos \omega t
$$

should be a very good representation of the periodic solution for arbitrary dimension $n$ of this system.

Let us define the equivalent gain in the $k$-th harmonic as $F_{k}=y_{k} / x_{k}$. This 
function $F_{k}=y_{k} / x_{k}$ is known as the describing function of the nonlinearity $f$.

This first order approximation leads us to consider the following balance equations :

$$
\begin{aligned}
& E G(0)=\frac{y_{0}}{x_{0}} G(0)=1, \\
& F G(i \omega)=\frac{y_{1}}{x_{1}} G(i \omega)=1,
\end{aligned}
$$

where

$$
\begin{aligned}
& y_{0}=\frac{1}{2 \pi} \int_{0}^{2 \pi} f\left(x_{0}+x_{1} \cos t\right) d t, \\
& y_{1}=\frac{1}{\pi} \int_{0}^{2 \pi} f\left(x_{0}+x_{1} \cos t\right) \cos t d t .
\end{aligned}
$$

For a single loop control system, $E$ is frequency independent and the zero balance equation (3.1) gives us the mean value $x_{0}$ as a function of the amplitude $x_{1}$ of the oscillation. Then, the first balance equation (3.2) becomes a function of the amplitude $x_{1}$ and the frequency $\omega$, and it is possible to separate this $x_{1}-\omega$ relation :

$$
G(i \omega)=\frac{1}{F\left(x_{1}\right)}
$$

The $G$ - and $1 / F$-curves are studied in the complex plane. If they intersect then the balance conditions are satisfied and a periodic solution exists, otherwise the system cannot oscillate. For $f$ monotonically decreasing, it can be proved that $1 / F$ is a continuous negative real function, and the set of amplitudes is 
a connected compact set [16]. An intersection of $G(i \omega)-$ and $1 / F\left(x_{1}\right)$-curves will occur only if :

$$
\frac{1}{F_{\mathrm{Max}}} \leq G\left(i \omega_{c}\right) \leq \frac{1}{F_{\min }}
$$

where $\omega_{c}$ is the frequency, so that the $G(i \omega)$-curve first intersects the negative real axis. This $G(i \omega)$-curve is often called the Nyquist contour of the linear element.

\subsection{Functions $R$ and $R^{*}$}

Let us consider the nonlinear system (2.1) represented by a feedback connection of a linear time-invariant dynamical system and a nonlinear element $f\left(P_{n}\right)$, as shown in Fig. 2. We shall study the behaviour of this autonomous system, investigating the existence of periodic solutions.

Using the simultaneous loop balance conditions, we can construct numerically a function $\mathcal{R}$ of the reaction constants, where if $\mathcal{R}>1$ the system oscillates, and if $\mathcal{R} \leq 1$ there is no periodic solution. Comparing the value of $\mathcal{R}$ and the numerical solution by Runge-Kutta method via MATLAB software, we find that this technique is very accurate.

In our case (see [16] for technical details), the unique condition for an intersection $G\left(i \omega_{c}\right) \leq 1 / F_{\min }$ results in defining $\mathcal{R}$ as functions of the parameters $b_{i}$ and $\beta_{k}$ : 


$$
\mathcal{R}\left(b_{i}, \beta_{k}\right)=\frac{\left|F_{\min }\right|}{b_{1} \cdots b_{n}} \cdot\left|\frac{G\left(i \omega_{c}\right)}{G(0)}\right|
$$

so that $\mathcal{R}\left(b_{i}, \beta_{k}\right)>1$ is equivalent to this latter condition.

An algorithm is then possible to determine the existence of limit cycle.

In the sequel, let us denote $R \equiv \mathcal{R}\left(b_{i}, 0\right)$ when the parameters $\beta_{k}$ are zero, and $R^{*}$ otherwise. Let us note that the nonlinear block is the same for these cases. When we compare an isolated system and an associated system with identical parameters, except $\beta_{k}$, we can prove, in special cases, the inequalities $R^{*}<R$ and $\omega_{c}^{*} \leq \omega_{c}$, where the star ${ }^{*}$ superscript represents an associated system. We then observe an increasing stability in the sense that the amplitude and frequency both decrease.

\section{Analysis of oscillations}

For the analysis of the non-associated system, i.e. when the value of $\beta_{k}$ is always zero, we introduce the linear filter $G$ :

$$
G(p)=\left\{\prod_{k=1}^{n}\left(p+b_{k}\right)\right\}^{-1}
$$


where $p$ denotes the differentiation operator. After one or several couplings, the harmonic balance method leads us to consider the following filter:

$$
G^{*}(p)=\left[\prod_{k=1}^{n} \frac{\left(p+b_{k}\right)\left(p+\beta_{k}\right)}{\left(p+b_{k}\right)\left(p+\beta_{k}\right)+p \beta_{k}}\right] G(p) .
$$

\section{Proposition 1}

(i) $G(i \omega)=G^{*}(i \omega)$ if and only if:

$$
\omega=0 \quad \text { or } \quad \beta_{k}=0, \forall k=1, \ldots, n \text {; }
$$

(ii) The arguments of $G(i \omega)$ and $G^{*}(i \omega)$ verify the following formulae:

$$
\operatorname{Arg} G^{*}(i \omega)=\operatorname{Arg} G(i \omega)-\psi(\omega), \quad \psi(\omega)=\sum_{k=1}^{n} \psi_{k}(\omega)
$$

with

$$
\psi_{k}(\omega)=\operatorname{Arctan} \frac{\left(b_{k}+2 \beta_{k}\right) \omega}{b_{k} \beta_{k}-\omega^{2}}-\operatorname{Arctan} \frac{\left(b_{k}+\beta_{k}\right) \omega}{b_{k} \beta_{k}-\omega^{2}}
$$

(iii) The modulus of $G(i \omega)$ and $G^{*}(i \omega)$ verify the following estimation:

$$
\left|G^{*}(i \omega)\right| \leq|G(i \omega)| \quad \forall \omega>0
$$

Proof. The expressions for $G(i \omega)$ and $G^{*}(i \omega)$ are:

$$
\begin{aligned}
G(i \omega) & =\left(\prod_{k=1}^{n} \frac{1}{\left(\omega^{2}+b_{k}^{2}\right)^{1 / 2}}\right) \exp \left(-i \sum_{k=1}^{n} \phi_{k}(\omega)\right) \\
G^{*}(i \omega) & =G(i \omega)\left(\prod_{l=1}^{n} \frac{\left(\omega^{2}+b_{l}^{2}\right)^{1 / 2}\left(\omega^{2}+\beta_{l}^{2}\right)^{1 / 2}}{\left(\omega^{2}+c_{l}^{2}\right)^{1 / 2}\left(\omega^{2}+d_{l}^{2}\right)^{1 / 2}}\right) \exp \left(-i \sum_{l=1}^{n} \psi_{l}(\omega)\right)
\end{aligned}
$$


with

$$
\phi_{k}(\omega)=\operatorname{Arctan} \frac{\omega}{b_{k}}
$$

and

$$
\begin{aligned}
\psi_{l}(\omega) & =\operatorname{Arctan} \frac{\omega}{c_{l}}+\operatorname{Arctan} \frac{\omega}{d_{l}}-\operatorname{Arctan} \frac{\omega}{b_{l}}-\operatorname{Arctan} \frac{\omega}{\beta_{l}} \quad \text { if } \beta_{l} \neq 0, \\
\psi_{l}(\omega) & =\phi_{l}(\omega) \text { if } \beta_{l}=0, \\
c_{l} & =\frac{\left(b_{l}+2 \beta_{l}\right)-\sqrt{b_{l}^{2}+4 \beta_{l}^{2}}}{2}>0, \\
d_{l} & =\frac{\left(b_{l}+2 \beta_{l}\right)+\sqrt{b_{l}^{2}+4 \beta_{l}^{2}}}{2}>0 .
\end{aligned}
$$

Let us consider the linear filters $G$ and $G^{*}$ with a unique coupling, i.e. with $\beta_{l}=\beta>0$ and $\beta_{j}=0$ for all $j, j \neq l$. The expressions of $G(i \omega)$ and $G^{*}(i \omega)$ are deduced from the above, and the equality $G(i \omega)=G^{*}(i \omega)$ leads to:

$$
\left(\omega^{2}+b_{l}^{2}\right)\left(\omega^{2}+\beta^{2}\right)=\left(\omega^{2}+c_{l}^{2}\right)\left(\omega^{2}+d_{l}^{2}\right) \quad \text { and } \quad \psi_{l}(\omega)=0,
$$

equivalent to:

$$
\omega=0 \quad \text { or } \quad \beta=0 .
$$

Moreover, since we have: $\psi_{l}(0)=0, \lim _{\beta \rightarrow 0^{+}} \psi_{l}(\omega)=0$, and $b_{l} \beta=c_{l} d_{l}$ when $\omega=0$ or $\beta=0$, the converse is obvious.

The arguments of $G(i \omega)$ and $G^{*}(i \omega)$ verify: $\operatorname{Arg} G^{*}(i \omega)=\operatorname{Arg} G(i \omega)-\psi_{l}(i \omega)$ and, by means of Arctan property:

$$
\psi_{l}(\omega)=\operatorname{Arctan} \frac{\left(b_{l}+2 \beta\right) \omega}{b_{l} \beta-\omega^{2}}-\operatorname{Arctan} \frac{\left(b_{l}+\beta\right) \omega}{b_{l} \beta-\omega^{2}} .
$$


The identities: $c_{l}^{2}+d_{l}^{2}=b_{l}^{2}+4 \beta^{2}+2 b_{l} \beta$, and $c_{l} d_{l}=b_{l} \beta$ lead to the inequality:

$$
\forall \omega \in \mathbb{R},\left|G^{*}(i \omega)\right| \leq|G(i \omega)|
$$

By successive iterations, we obtain the same results with multiple couplings.

Corollary 2 In the complex plane, the $G^{*}(i \omega)$-curve, for $\omega>0$, is nearer the origin than the $G(i \omega)$-curve.

Proof. Let us note that we have: $G^{*}(0)=G(0) \in \mathbb{R}^{*+}$, and $\forall \omega>0$, $\operatorname{Arg} G(i \omega)=-\sum_{k=1}^{n} \operatorname{Arctan} \frac{\omega}{b_{k}}<0$. If $\omega$ is large enough, the Arctan property gives us $\psi_{l}(\omega)<0$, then $\operatorname{Arg} G^{*}(i \omega)>\operatorname{Arg} G(i \omega)$. Moreover, as positive $\omega$ increases, the modulus and the argument of $G(i \omega)$ and $G^{*}(i \omega)$ decrease monotonically:

$$
\begin{gathered}
\lim _{\omega \rightarrow+\infty} G(i \omega)=\lim _{\omega \rightarrow+\infty} G^{*}(i \omega)=0, \\
\lim _{\omega \rightarrow+\infty} \operatorname{Arg} G(i \omega)=\lim _{\omega \rightarrow+\infty} \operatorname{Arg} G^{*}(i \omega)=-n \frac{\pi}{2} .
\end{gathered}
$$

The curves spiral towards the origin. Since the $G^{*}(i \omega)$-curve only intersects the $G(i \omega)$-curve when $\omega=0$ or when $\beta_{k} \equiv 0$, for all $k$, and since the modulus and the arguments of $G^{*}(i \omega)$ are smaller than those of $G(i \omega)$ when $\omega$ is of a sufficiently large value, we therefore deduce that the $G^{*}(i \omega)$-curve is nearer the origin in the complex plane than the $G(i \omega)$-curve. 
The $G(i \omega)$ - and $G^{*}(i \omega)$-curves first cross the negative real axis at frequencies $\omega_{c}$ and $\omega_{c}^{*}$, if they exist, so that:

$$
\begin{aligned}
\sum_{i=1}^{n} \operatorname{Arctan} \frac{\omega_{c}}{b_{i}} & =\pi \\
\sum_{i=1}^{n}\left(\operatorname{Arctan} \frac{\omega_{c}^{*}}{b_{i}}+\psi_{i}\left(\omega_{c}^{*}\right)\right) & =\pi
\end{aligned}
$$

where

$$
\psi_{i}\left(\omega_{c}^{*}\right)=\operatorname{Arctan} \frac{\left(b_{i}+2 \beta_{i}\right) \omega_{c}^{*}}{b_{i} \beta_{i}-\left(\omega_{c}^{*}\right)^{2}}-\operatorname{Arctan} \frac{\left(b_{i}+\beta_{i}\right) \omega_{c}^{*}}{b_{i} \beta_{i}-\left(\omega_{c}^{*}\right)^{2}} .
$$

This expression can be further simplified when the following special case is considered. Let us note that a solution $\omega_{c}>0$ of Eq. (4.1) exists if and only if $n \geq 3$.

\section{Proposition 3}

(i) If $\beta_{l} b_{l}>\omega_{c}^{2}$ for all $l=1, \ldots, n$, then $\omega_{c}^{*} \leq \omega_{c}$;

(ii) If $b_{k} \equiv b$ and $\beta_{k} \equiv \beta>0, \quad 1 \leq k \leq n$, then

(a) $\omega_{c}^{*} \leq \omega_{c} \quad$ if and only if $\quad \beta \geq b \tan ^{2} \frac{\pi}{n}$.

(b) $R^{*}<R$.

Proof. If $\beta_{l} b_{l}>\omega_{c}^{2}$, for all $l=1, \ldots, n$, then we have:

$$
\psi_{l}\left(\omega_{c}\right)>0, \quad \text { and } \quad \sum_{l=1}^{n}\left(\operatorname{Arctan} \frac{\omega_{c}}{b_{l}}+\psi_{l}\left(\omega_{c}\right)\right)>\pi
$$

so we obtain: $\omega_{c}^{*} \leq \omega_{c}$. 
If $\quad b_{k} \equiv b$ and $\beta_{k} \equiv \beta>0,1 \leq k \leq n$, we obtain first: $\omega_{c}=b \tan \frac{\pi}{n}$, and we consider the formula (4.2):

$$
\sum_{l=1}^{n} \operatorname{Arctan} \frac{\omega_{c}^{*}}{b}+\psi_{l}\left(\omega_{c}^{*}\right)=\pi
$$

Let us note that $\psi_{l}\left(\omega_{c}\right)=0$ according to the case $\beta=0$.

Let us denote $\psi \equiv n \psi_{0}$ with

$$
\psi_{0}(\omega)=\operatorname{Arctan} \frac{(b+2 \beta) \omega}{b \beta-\omega^{2}}-\operatorname{Arctan} \frac{(b+\beta) \omega}{b \beta-\omega^{2}} .
$$

We observe that, under this form, $\psi_{0}(\omega)=0$ if and only if $\omega=0$, or $\sqrt{b \beta}$, or $+\infty$ to the limit. In $\mathbb{R}^{+}$, the function $\psi_{0}$ is positive in the interval $[0, \sqrt{b \beta}]$.

Let us denote $\omega_{0}^{*}=\sqrt{b \beta}$. If $\beta \geq b \tan ^{2} \frac{\pi}{n}$, then $\forall \omega \in[0, \sqrt{b \beta}], \psi_{0}>0$, and $n \operatorname{Arctan} \frac{\omega_{0}^{*}}{b} \geq \pi$, this implies: $\omega_{c}^{*} \leq \omega_{0}^{*}$.

Since $n \psi_{0}\left(\omega_{c}^{*}\right) \geq 0$, we can deduce: $n \operatorname{Arctan} \frac{\omega_{c}^{*}}{b}<\pi$, this implies: $\omega_{c}^{*} \leq \omega_{c}$.

Conversely, if $\omega_{c}^{*} \leq \omega_{c}$, then $\omega_{c}^{*} \leq b \tan \frac{\pi}{n}$, and

$$
\sum_{l=1}^{n} \operatorname{Arctan} \frac{\omega_{c}^{*}}{b_{l}}=n \operatorname{Arctan} \frac{\omega_{c}^{*}}{b} \leq \pi
$$

this leads to: $\psi\left(\omega_{c}^{*}\right) \geq 0$, then $\omega_{c}^{*} \in[0, \sqrt{b \beta}]$.

Secondly, it is sufficient to prove $\left|G^{*}\left(i \omega_{c}^{*}\right)\right|<\left|G\left(i \omega_{c}\right)\right|$. The representation of $G^{*}$ leads to:

$$
\left|G^{*}(i \omega)\right|<|G(i \omega)| \quad \forall \omega>0 \text {. }
$$


The functions $\left|G^{*}(i \omega)\right|$ and $|G(i \omega)|$ versus $\omega$ are continuous and monotonically decreasing with increasing positive frequency.

Let us denote $\omega_{d}$ so that $\left|G^{*}\left(i \omega_{c}^{*}\right)\right|=\left|G\left(i \omega_{d}\right)\right|$.

If $\omega_{c}<\omega_{d},\left|G\left(i \omega_{d}\right)\right|<\left|G\left(i \omega_{c}\right)\right|$, then $\left|G^{*}\left(i \omega_{c}^{*}\right)\right|<\left|G\left(i \omega_{c}\right)\right|$.

It remains to prove: $\omega_{c}<\omega_{d}$, so $\left|G^{*}\left(i \omega_{c}^{*}\right)\right|<\left|G\left(i \omega_{c}\right)\right|$ is always verified. The equality:

$$
\operatorname{Arctan} \frac{\omega_{c}^{*}}{b}+\operatorname{Arctan} \frac{(b+2 \beta) \omega_{c}^{*}}{b \beta-\left(\omega_{c}^{*}\right)^{2}}-\operatorname{Arctan} \frac{(b+\beta) \omega_{c}^{*}}{b \beta-\left(\omega_{c}^{*}\right)^{2}}=\operatorname{Arctan} \frac{\omega_{c}}{b}
$$

leads to:

$$
\omega_{c}=\frac{b \omega_{c}^{* 5}+\left(b^{3}+2 b \beta^{2}\right) \omega_{c}^{* 3}+2 b^{3} \beta^{2} \omega_{c}^{*}}{(b+\beta) \omega_{c}^{* 4}+\left(b^{2} \beta+b^{3}+b \beta^{2}\right) \omega_{c}^{* 2}+b^{3} \beta^{2}} .
$$

Moreover, $\left|G\left(i \omega_{d}\right)\right|=\left|G^{*}\left(i \omega_{c}^{*}\right)\right|$ implies:

$$
\omega_{d}^{2}=\frac{\omega_{c}^{* 4}+\left(2 b \beta+4 \beta^{2}\right) \omega_{c}^{* 2}}{\omega_{c}^{* 2}+\beta^{2}} .
$$

So, we obtain:

$$
\omega_{d}^{2}-\omega_{c}^{2}=C_{1} \omega_{c}^{* 12}+C_{2} \omega_{c}^{* 10}+C_{3} \omega_{c}^{* 8}+C_{4} \omega_{c}^{* 6}+C_{5} \omega_{c}^{* 4}+C_{6} \omega_{c}^{* 2}
$$

with

$$
\begin{aligned}
& C_{1}=\beta^{2}+2 b \beta>0, C_{2}=6 b^{3} \beta+12 b \beta^{3}+6 b^{2} \beta^{2}+4 \beta^{4}>0, \\
& C_{3}=\left(4 b^{3} \beta+2 b \beta^{3}\right)\left(2 b \beta+4 \beta^{2}\right)+12 b^{3} \beta^{3}+9 b^{2} \beta^{4}+6 b^{5} \beta+3 b^{4} \beta^{2}>0, \\
& C_{4}=2\left(b^{4} \beta^{2}+b^{3} \beta^{3}\right)\left(2 b \beta+4 \beta^{2}\right)+2 b^{3} \beta^{2}\left(b^{2} \beta+b \beta^{2}+b^{3}\right)+10 b^{3} \beta^{5}+10 b^{5} \beta^{3}+ \\
& 3 b^{6} \beta^{2}+2 b^{7} \beta>0, \\
& C_{5}=5 b^{6} \beta^{4}+10 b^{5} \beta^{5}+4 b^{7} \beta^{3}>0 \quad \text { and } C_{6}=2 b^{7} \beta^{5} .
\end{aligned}
$$


Lastly, we deduce: $\omega_{c}<\omega_{d}$, then $R^{*}<R$.

\section{Applications}

(i) According to the condition: $\beta \geq b \tan ^{2} \frac{\pi}{n}$, when $n \geq 3$, it is possible to find $\omega_{c}$ and $\omega_{c}^{*}$ solutions of Eqs (4.1) and (4.2). This condition leads to $\beta \geq+\infty$ when $n=2, \beta \geq 3 b$ when $n=3$, and $\beta \geq b / 3$ when $n=6$. In the case $n=2$, when $b_{k} \equiv b$ and $\beta_{l} \equiv \beta$, the formulae (4.1) and (4.2) have no solutions: no oscillations occur. In the cases $n>2$, couplings with large $\beta$ give smaller frequencies.

(ii) The steady states of systems with or without coupling(s) give identical concentrations $P_{i}, 1 \leq i \leq n$, and when steady state is unstable, oscillations appear [14] and coupling decreases the frequency of the oscillations of the system if the diffusion coefficient $\beta$ is of a large enough value versus kinetic coefficient $b$. In addition, if the number of reactants increases, then the stability effect of pools is more effective:

$$
n \rightarrow+\infty \Longrightarrow \beta \geq b \tan ^{2} \frac{\pi}{n} \rightarrow 0 \Longrightarrow \omega_{c}^{*} \leq \omega_{c}
$$

\section{Proposition 4}

If $b_{k}=\beta_{k} \equiv b$, for all $k$, then

(i) $n=4 \Longrightarrow \omega_{c}^{*}=\omega_{c}$;

(ii) $n>4 \Longrightarrow \omega_{c}^{*} \leq \omega_{c}$. 
Proof. If $b_{k}=\beta_{k} \equiv b, \quad \forall k$, then we have:

$$
\psi_{0}(\omega)=\operatorname{Arctan} \frac{2 \omega}{(3-\sqrt{5}) b}+\operatorname{Arctan} \frac{2 \omega}{(3+\sqrt{5}) b}-2 \operatorname{Arctan} \frac{\omega}{b}
$$

The expression of $\psi_{0}\left(\omega_{c}\right)$ gives us:

- if $n=4, \operatorname{Arctan} \frac{2}{3+\sqrt{5}}+\operatorname{Arctan} \frac{2}{3-\sqrt{5}}=\frac{\pi}{2}$, then $\omega_{c}^{*}=\omega_{c}$;

- if $n \geq 4$, then $\psi_{0}\left(\omega_{c}\right)=\operatorname{Arctan} \frac{3 \tan \pi / n}{1-\tan ^{2} \pi / n}-\frac{2 \pi}{n} \geq 0$, and the result is obtained, i.e.: $\omega_{c}^{*} \leq \omega_{c}$.

\section{Proposition 5}

Let us assume that the systems admit both oscillations, i.e. the $G(i \omega)$ - and $1 / F(x)$-curves intersect in the complex plane at point $(-x ; 0)$ of negative real axis (see [16]), and the $G^{*}(i \omega)$ - and $1 / F\left(x^{*}\right)$-curves intersect at point $\left(-x^{*} ; 0\right)$ (so, we consider the amplitudes $\left.x, x^{*}>0\right)$.

Then: $x^{*}<x$.

Proof. The above results state that, since $\left|G^{*}(i \omega)\right| \leq|G(i \omega)|$, the $G^{*}(i \omega)$ curve is nearer the origin in the complex plane than the $G(i \omega)$-curve. The $G^{*}(i \omega)$-curve spirals towards the origin in a clockwise direction faster than the $G(i \omega)$-curve. This is sufficient to prove the above result. Indeed, when we consider the associated system, the balance method gives us the following first harmonic equation: $G^{*}(i \omega)=1 / F(x)$ where $F$ is a real function of $x$, the amplitude of the oscillation, and $\omega$, the frequency (coupling does not modify 
this function $F)$. If the $G(i \omega)-$, respectively $G^{*}(i \omega)-$, and $1 / F(x)$-curves intersect in the complex plane, oscillations occur with amplitude $x$, respectively $x^{*}$, and the amplitude $x^{*}$ of a periodic solution is smaller when coupling exists (the point $-x^{*}$ of the negative real axis is nearer the origin than the point $-x)$.

\section{$5 \quad$ Numerical experiments}

Let us compute the expressions $R$ and $R^{*}$. First of all, we need the expressions of $G$ and $G^{*}$, and then the approximate solutions $\omega_{c}$ and $\omega_{c}^{*}$ of Eqs (4.1) and

Simulations are obtained using the original system:

$$
\begin{cases}\frac{d S_{1}}{d \tau}=\frac{\alpha_{0}}{1+K S_{n}^{\mu}}-\alpha_{1} S_{1}-g_{1}\left(S_{1}-S_{1}^{*}\right), & \\ \frac{d S_{k}}{d \tau}=\alpha_{k-1} S_{k-1}-\alpha_{k} S_{k}-g_{k}\left(S_{k}-S_{k}^{*}\right), & k=2, \ldots, n, \\ \frac{d S_{k}^{*}}{d \tau}= & g_{k}\left(S_{k}-S_{k}^{*}\right), \quad k=1, \ldots, n .\end{cases}
$$

Defining new variables $t, P_{1}, \ldots, P_{n}, P_{1}^{*}, \ldots, P_{n}^{*}$ as:

$$
\begin{aligned}
t & =\left(\alpha_{1} \cdots \alpha_{n-1} K^{1 / \mu} \alpha_{0}\right)^{1 / n} \tau, \\
P_{1} & =\alpha_{0}^{-1}\left(\alpha_{1} \cdots \alpha_{n-1} K^{1 / \mu} \alpha_{0}\right)^{1 / n} S_{1},
\end{aligned}
$$


Table 1

Non-associated system: $\alpha_{j}=1, \quad j=1, \ldots, n-1, \mu=5$

\begin{tabular}{r|r|r|r|r|l}
\hline$n$ & \multicolumn{1}{|c|}{$\alpha_{0}$} & \multicolumn{1}{c|}{$K$} & $\alpha_{n}$ & $R$ & Oscillation \\
\hline 5 & 50 & 0.01 & 0.5 & 0.8673 & No \\
5 & 50 & 1 & 1 & 0.9851 & No \\
7 & 5 & 0.1 & 0.5 & 0.9730 & No \\
7 & 50 & 1 & 5 & 1.1115 & Yes \\
8 & 5 & 0.1 & 0.5 & 1.0811 & Yes \\
8 & 5 & 0.1 & 1 & 0.8653 & No \\
\hline
\end{tabular}

$$
\begin{aligned}
& P_{1}^{*}=\alpha_{0}^{-1}\left(\alpha_{1} \cdots \alpha_{n-1} K^{1 / \mu} \alpha_{0}\right)^{1 / n} S_{1}^{*}, \\
& P_{k}=\left(\alpha_{1} \cdots \alpha_{k} \alpha_{0}\right)^{-1}\left(\alpha_{1} \cdots \alpha_{n-1} K^{1 / \mu} \alpha_{0}\right)^{k / n} S_{k}, \quad k=2, \ldots, n, \\
& P_{k}^{*}=\left(\alpha_{1} \cdots \alpha_{k} \alpha_{0}\right)^{-1}\left(\alpha_{1} \cdots \alpha_{n-1} K^{1 / \mu} \alpha_{0}\right)^{k / n} S_{k}^{*}, \quad k=2, \ldots, n .
\end{aligned}
$$

the resulting equations are equations (2.1) where:

$$
b_{k}=\alpha_{k}\left(\alpha_{1} \cdots \alpha_{n-1} K^{1 / \mu} \alpha_{0}\right)^{-1 / n}, \quad \beta_{k}=g_{k}\left(\alpha_{1} \cdots \alpha_{n-1} K^{1 / \mu} \alpha_{0}\right)^{-1 / n} .
$$

\subsection{Non-associated system}

Simulations are obtained, and results are shown in Table 1 using the original system (5.1). Direct simulations using MATLAB software confirm this fact. 
Table 2

\begin{tabular}{rr|r|r|r|l} 
Associated system: $\alpha_{j}=1$, & $j=1, \ldots, n-1, g_{l}=1, \quad l=1, \ldots, n, \quad \mu=3$ \\
\hline$n$ & $\alpha_{0}$ & $K$ & $\alpha_{n}$ & $R^{*}$ & Oscillation \\
\hline $\begin{array}{r}7 \\
8\end{array}$ & 50 & 0.1 & 0.5 & 0.8735 & No \\
9 & 10 & 10 & 1 & 0.9953 & No \\
10 & 50 & 0.1 & 0.5 & 1.2199 & Yes \\
12 & 1 & 2 & 0.5 & 0.8911 & No \\
15 & 200 & 0.01 & 0.1 & 1.2414 & Yes \\
\hline
\end{tabular}

\subsection{Associated system}

Some results are shown in Table 2, where the existence of oscillations is pointed out.

Comparing the value of $R^{*}$ and the numerical solution of the set of equations (5.1), we observe that the values of $R^{*}$ confirm or deny the existence of a periodic solution. 
Table 3

Isolated and associated systems:

\begin{tabular}{|c|c|c|c|c|c|}
\hline$n$ & $\alpha_{0}$ & $K$ & $\alpha_{n}$ & $R$ & $R^{*}$ \\
\hline 7 & 50 & 0.1 & 0.5 & 1.2949 & 0.8735 \\
\hline 8 & 5 & 0.1 & 1 & 0.8653 & 0.5992 \\
\hline 9 & 150 & 1 & 1 & 1.6742 & 1.2231 \\
\hline 10 & 10 & 2 & 0.1 & 0.9513 & 0.7967 \\
\hline 12 & 100 & 0.01 & 5 & 1.3426 & 1.0568 \\
\hline
\end{tabular}

5.3 Comparison between isolated and associated systems

The results in Table 3 exhibit the effects of coupling on the value $R^{*}$ versus $R$. When $g_{k}$ is not equal to zero, the numerical values of $R^{*}$ are always smaller than those of $R$. The stability of associated systems is increased.

\subsection{Frequency comparison}

We here assume: $\alpha_{j}=a, \quad j=1, \ldots, n$, and $g_{k}=g, \quad k=1, \ldots, n$. Let us note that the condition $\beta>b \tan ^{2} \frac{\pi}{n}$ for the nondimensioned system (2.1) is equivalent to the condition $g>a \tan ^{2} \frac{\pi}{n}$ for the original system (5.1). We can 
Table 4

Frequency comparison: $\alpha_{j}=a, \quad j=1, \ldots, n, g_{l}=g, \quad l=1, \ldots, n, \quad K=2$

\begin{tabular}{c|c|c|c|c|c|c}
\hline$n$ & $\alpha_{0}$ & $\mu$ & $g$ & $a \tan ^{2} \frac{\pi}{n}$ & $\omega_{c}$ & $\omega_{c}^{*}$ \\
\hline 3 & 50 & 6 & 0.065 & 0.783 & 0.452 & 0.556 \\
4 & 200 & 7 & 0.046 & 0.461 & 0.461 & 0.502 \\
4 & 50 & 5 & 0.726 & 0.363 & 0.363 & 0.222 \\
6 & 5 & 6 & 0.210 & 0.280 & 0.486 & 0.516 \\
6 & 200 & 2 & 1.020 & 0.170 & 0.294 & 0.155 \\
8 & 100 & 4 & 1.009 & 0.086 & 0.209 & 0.107 \\
\hline
\end{tabular}

verify the property $\omega_{c}^{*}<\omega_{c}$ when this condition is satisfied (see, for instance,

Table 4 where the opposite cases are shown). In these cases, we have also obtained $R^{*}<R$. Extensive simulations have directed us to the special case: if $b_{k} \equiv b, \beta_{k} \equiv \beta$, for all $k=1, \ldots, n$, so that $b=\beta \tan ^{2} \frac{\pi}{n}$, then $n=22$ has given $\omega_{c}^{*}=\omega_{c}$.

\section{Discussion}

Models of Yates-Pardee-Goodwin metabolic pathway in a homogeneous milieu can especially display stable steady states or limit cycle oscillations, depending on the parameter values. On one hand, stable steady states can be viewed 
as "homeostatic behaviour". On the other hand, the physiological significance of biochemical oscillations, if they do occur in vivo, may be varying, and is somewhat open for speculation (for a review, see [6]). For instance, biochemical oscillations may be involved in signal transmission, excitability, specification of positional information during, e.g., embryogenesis. However, it was also suggested that undamped oscillations may sometimes be an "undesirable phenomenon under circumstances where a steady state (homeostatic behaviour) is highly desirable" (see e.g. [18]).

Recently, a great deal of attention has been paid to the heterogeneity and anisotropy of the cell milieu. More precisely, the spatial localization of enzymes for specific metabolic processes, which is often referred to as enzyme organization, may be due to $(i)$ segregation of related metabolic pathways in organelles, (ii) adsorption of enzymes to membranes or cytoskeleton or (iii) multienzyme complexes resulting in molecular "channelling" [21]. Although the existence of cellular microenvironments has been demonstrated experimentally in many cases, the physiological significance of cellular enzyme organization remains open for speculation, especially as little is known about the consequences of the existence of compartments on the dynamic behaviour of metabolic pathways.

Since the parallel association of metabolic chains can result in an increase in their stability domain $[1,2,14]$, we have studied, in the present work, some effects of coupling by diffusion in a system with enzyme organization on the 
oscillations of a Yates-Pardee-Goodwin metabolic chain.

In our model, the Yates-Pardee-Goodwin pathway is confined to the so-called interior compartment, while exchange of metabolites can occur between the interior and the exterior compartment. Owing to the great ubiquity of the YatesPardee-Goodwin metabolic chains [13], this scheme is likely to be encountered in many instances in cell metabolism. For instance, the enzymes of Krebs cycle are confined to the mitochondria matrix, and metabolites such that malate or $\alpha$-ketoglutarate can be exchanged with cytosol. Moreover, single loop negative feedback do exist in Krebs cycle, e.g.: $(i)$ succinate-dehydrogenase is inhibited by oxaloacetate, $(i i)$ citrate-synthetase is inhibited by succinyl-CoA. Although the exchanges of metabolites between cytosol and mitochondria are generally more complex than simple diffusion, our proposed scheme may be considered as a paradigm of this sort of situation, owing to the robustness of the obtained results.

Our results show that coupling with exterior pools of metabolites modifies the behaviour of the oscillations of a Goodwin metabolic system in the following way: $(i)$ periodic solutions of the system with coupling have lower amplitudes and frequencies; (ii) coupling can give rise to a steady state, instead of a periodic solution. These observations are consistent with the increase of stability observed when two Yates-Pardee-Goodwin metabolic pathways are associated [14]. Thus the stability of the Goodwin-Yates-Pardee metabolic pathway, which is the most commonly encountered form of a regulated biochemical 
pathway, is enhanced when matter can be exchanged with that outside. These results suggest that the presence of cellular microenvironments can result in a diversity of metabolic dynamics, either homeostatic (asymptotically stable steady state) or oscillatory, depending on the exchanges between the microenvironment and the exterior.

\section{Acknowledgement}

The authors are grateful to the Conseil général du Maine-et-Loire and the Laboratoire d'Automatique FSSM Marrakech for supporting this research. 


\section{References}

[1] Chauvet G.A., Hierarchical functional organization of formal biological systems: a dynamical approach. I. The increase in complexity by self-association increases the domain of stability of a biological system. Phil. Trans. R. Soc. Lond. B 339 (1993) 425-444.

[2] Chauvet G. A. and Costalat R., On the functional organization in a biological structure: the example of enzyme organization. C.R.Acad.Sci. Paris, Sciences de la vie / Life Sciences 318 (1995) 529-535.

[3] Costalat R. and Burger J., Effect of enzyme organization on the stability of Yates-Pardee pathways. Bull. Math. Biol. in press.

[4] Gantmakher F. R., The Theory of Matrices (2 vols, translated by K.A. Hirsch. Chelsea Pub. Co, New York, 1959).

[5] Gille J.-Ch., Systèmes Asservis Non Linéaires (Dunod, Paris, 1988).

[6] Goldbeter A., Rythmes et Chaos dans les Systèmes Biochimiques et Cellulaires (Masson, Paris, 1990).

[7] Goodwin B.C., Analytical Physiology of Cells and Developing Organisms (Academic Press, London, 1976).

[8] Hastings S., Tyson J. and Webster D., Existence of periodic solutions for negative feedback cellular control systems. Journal of Diff. Eq. 25 (1977) 39-64. 
[9] Khalil H. K., Nonlinear Systems (Macmillan Publishing Company, New York, 1992).

[10] Marmillot P., Hervagault, J.-F. and Welch G. R., Patterns of spatiotemporal organization in an "ambiquitous" enzyme model. Proc. Natl. Acad. Sci. USA, Biochemistry 89 (1992) 12103-12107.

[11] Mees A., The describing function matrix. J. Inst. Maths. Applics. 10 (1972) 49-67.

[12] Mees A., Limit cycle stability. J. Inst. Maths. Applics. 11 (1973) 281-295.

[13] Monod J., Changeux J.-P. and Jacob F., Allosteric proteins and cellular control systems. J. Mol. Biol. 6 (1963) 306-329.

[14] Morillon J.-P., Costalat R., Burger N. and Burger J., Modelling two associated biochemical pathways. In Proc. of the IMACS Symposium on Mathematical Modelling; 1. MathMod Vienna 3 (Vienna, Austria, Technical University Vienna, 1994) pp. 391-394.

[15] Rapp P., Analytic procedures for large dimension nonlinear biochemical oscillators. BioSystems 7 (1975) 92-100.

[16] Rapp P., Analysis of biochemical phase shift oscillators by a harmonic balancing technique. J. Math. Biol. 3 (1976) 203-224.

[17] Tyson J. J. and Othmer H. G., The Dynamics of Feedback Control Circuits in Biochemical Pathways. Progress in Theoretical Biol. 5 (Academic Press, New York, 1978). 
[18] Viniegra-Gonzalez G., Stability properties of metabolic pathways with feedback interactions. In Biological and Biochemical Oscillators, Proc. of Two Symposia: Prague, Czechoslovakia, July 19-21, 1968, and Hango, Finland, August 16-17, 1969, ed. by Change, Britton et al. (Academic Press, New York, 1973) pp. 41-59.

[19] Walter C.F., The absolute stability of certain types of controlled biological systems. J. Theor. Biol. 23 (1969) 39-52.

[20] Walter C.F., Kinetic and thermodynamic aspects of biological and biochemical control mechanisms. In Biochemical Regulatory Mechanisms in Eukaryotic Cells, ed. by Kun Ernest and Santiago Grisolia (Wiley-Interscience, 1972) pp. 355-489.

[21] Westerhoff H.V. and Welch G.R., Enzyme organization and the direction of metabolic flow: physicochemical considerations. Current Topics in Cellular Regulation 23 (1992) 361-390. 